\title{
THE TRANSVERSE VIBRATIONS OF A PIPE CONTAINING FLOWING FLUID: METHODS OF INTEGRAL EQUATIONS*
}

\author{
BY \\ LOUISE H. JONES AND BRUCE E. GOODWIN
}

University of Delaware

\begin{abstract}
Methods are developed to study the problem described in the title. Improvable lower bounds for the first eigenvalue are obtained for the low velocity-thin pipe wall case. It is shown that the eigenvalue changes from real to imaginary as the fluid velocity increases through a "critical" velocity. It is the methods which we wish to emphasize in that while we discuss them only for the present problem they are very general and especially powerful when applied to differential equations with constant coefficients.
\end{abstract}

1. Introduction. The problem we wish to consider reduces to the study of the eigenvalue problem for the differential equation

$$
u^{\mathrm{IV}}+k v^{2} u^{\mathrm{II}}+i v \beta u^{\mathrm{I}}-\beta^{2} u=0
$$

together with appropriate boundary conditions. At first glance one might think that the problem is not very difficult in that the differential equation has constant coefficients. However, the problem possesses several difficulties: (1) the problem is not self-adjoint; (2) one coefficient is imaginary; and (3) the operator is a nonlinear function of the eigenvalue parameter $\beta$. The first two difficulties rule out the possibility of a variational approach to the problem, as can be proven using the results of [2]. The third difficulty is formidable and represents the novel feature of the problem.

The present paper is concerned with the method of integral equations only formally different from the methods of [3].

From the symmetrization [9, pp. 145-150] of Green's function for the low velocity case, one can deduce the existence of an infinite number of real eigenvalues from the theorems of Iglisch [4]. In the high velocity case, Green's function is a classical Schmidt kernel and hence has only real eigenvalues, $\beta^{2}$. We assume the existence of eigenvalues in the general case; to prove this assumption is by no means a simple matter.

We concern ourselves here with a simply supported pipe but the method applies to any of the boundary conditions for beams.

2. Historical background. The problem of determining the lowest frequency of vibration of a pipe containing flowing fluid was first studied in connection with vibration of the Trans-Arabian Oil Pipeline. The partial differential equation governing the transverse vibration of a pipe considered as a beam was derived from Hamilton's prin-

*Received February 27, 1969; revised version received August 10, 1970. Portions of this work form part of the thesis [1]. Both authors are indebted to the National Science Foundation and the University of Delaware Research Foundation for financial support. 
ciple by Housner [5]. The equation was separated assuming harmonic time dependence, $e^{i \beta t}$, and put into the following nondimensional form by Long [6]:

$$
u^{\mathrm{IV}}+k v^{2} u^{\mathrm{II}}+i v \beta u^{\mathrm{I}}-\beta^{2} u=0,
$$

where $u$ is the transverse deflection, $v$ is the dimensionless flow velocity, and $k$ is a constant essentially determined by the geometry of the tube. Roman numerals indicate differentiation with respect to $x(0<x<1)$, and $\beta$ is the eigenvalue. Niordson [10], who studied the vibration of the plenstock tubes of the Aswan Dam, also derived (1) from shell theory as the special case of a tube whose cross-section is unchanged in the deformed state (i.e., a tube which vibrates as a beam).

Conventional power series solutions for (1) were investigated by Ashley and Haviland [7] and Long [6]. Their work showed that it was necessary to use extreme care in evaluating the resulting high-order determinants in order to obtain correct results. Long found that flowing fluid caused a small decrease in the frequency of vibration but no damping. Handelman [8] used perturbation techniques to determine the behavior of the eigenvalues without first solving the differential equation. He found that at low fluid velocity the eigenvalue remained real and decreased slightly as the velocity increased, while at high fluid velocity the eigenvalue approached zero and then became complex as the velocity increased. Thus, Handelman concluded that above a certain "critical" velocity the tube will be unstable due to exponential time dependent terms. Handelman's results also agreed with those of Niordson.

The purpose of this paper is to describe methods of integral equations which provide improvable lower bounds for the eigenvalues of differential equations which are nonlinear in the eigenvalue parameter.

3. Integral equation formulation. The transverse vibrations of a simply supported pipe containing flowing fluid are described by the differential equation (1) together with the boundary conditions

$$
u(0)=u^{\mathrm{II}}(0)=u(1)=u^{\mathrm{II}}(1)=0,
$$

or by the equivalent integral equation:

$$
u(y)=-\beta^{2} \int_{0}^{1} G(x, y, \beta) u(x) d x .
$$

$G(x, y, \beta)$ is Green's function and is given by:

$$
\begin{aligned}
G(x, y, \beta) & =G_{1}(x, y, \beta), & & 0 \leq x<y \leq 1, \\
& =G_{2}(x, y, \beta), & & 0 \leq y<x \leq 1,
\end{aligned}
$$

where $G_{1}$ and $G_{2}$ satisfy the adjoint equation

$$
\frac{\partial^{4} G}{\partial x^{4}}+k v^{2} \frac{\partial^{2} G}{\partial x^{2}}-i v \beta \frac{\partial G}{\partial x}=0,
$$

and the boundary conditions

$$
\begin{gathered}
G_{1}(0, y, \beta)=\frac{\partial^{2} G_{1}}{\partial x^{2}}(0, y, \beta)=0, \quad G_{2}(1, y, \beta)=\frac{\partial^{2} G_{2}}{\partial x^{2}}(1, y, \beta)=0 . \\
G(x, y, \beta), \frac{\partial G}{\partial x}(x, y, \beta), \quad \text { and } \frac{\partial^{2} G}{\partial x^{2}}(x, y, \beta) \text { are continuous at } x=y
\end{gathered}
$$


while

$$
\frac{\partial^{3} G_{1}}{\partial x^{3}}\left(y^{-}, y, \beta\right)-\frac{\partial^{3} G_{2}}{\partial x^{3}}\left(y^{+}, y, \beta\right)=1 .
$$

Eq. (2) follows from Green's formula:

$$
\int_{0}^{1}\left\{G(x, y, \beta) L_{x}(u)-u(x) \bar{L}_{x}(G(x, y, \beta))\right\} d x=\left.P(u, G)\right|_{0} ^{1}
$$

where

$$
L_{x}(u)=\frac{d^{4} u}{d x^{4}}+k v^{2} \frac{d^{2} u}{d x^{2}}+i v \beta \frac{d u}{d x}=+\beta^{2} u,
$$

$\bar{L}_{x}$ is the operator adjoint to $L_{x}$, and $P(u, G)$ is the bilinear concomitant of $u$ and $G$. Since $L_{x}=d^{4} / d x^{4}+k v^{2} d^{2} / d x^{2}+i v \beta d / d x$ is not self adjoint, $G(x, y, \beta)$ will not be symmetric in $x$ and $y$.

Now, $\bar{L}_{x}$ is a differential operator with constant coefficients so $G_{1}(x, y, \beta)=$ $\sum_{i-1}^{4} a_{i} e^{\alpha_{i x}}$ and $G_{2}(x, y, \beta)=\sum_{i=1}^{4} b_{i} e^{\alpha_{i x}}$ where $\alpha_{i}, i=1,2,3,4$, are roots of $\alpha^{4}+$ $k v^{2} \alpha^{2}-i v \beta \alpha=0$. Clearly $\alpha_{1}=0$ and $\alpha_{2}, \alpha_{3}$, and $\alpha_{4}$ are roots of the cubic $\alpha^{3}+k v^{2} \alpha-$ $i v \beta=0$. These roots can be determined by radicals, and will be linear combinations of

$$
\left(\frac{i v \beta}{2}+\left(-\frac{v^{2} \beta^{2}}{4}+\frac{k^{3} v^{6}}{27}\right)^{1 / 2}\right)^{1 / 3}
$$

and

$$
\left(\frac{i v \beta}{2}-\left(-\frac{v^{2} \beta^{2}}{4}+\frac{k^{3} v^{6}}{27}\right)^{1 / 2}\right)^{1 / 3}
$$

Calculations involving these radicals will be extremely cumbersome unless either $k^{3} v^{6} / 27$ can be neglected relative to $v^{2} \beta^{2} / 4$ or $v^{2} \beta^{2} / 4$ can be neglected relative to $k^{3} v^{6} / 27$. Thus, we consider two special cases:

$$
\text { (1) }\left|\frac{k^{3} v^{6}}{27}\right| \ll\left|\frac{v^{2} \beta^{2}}{4}\right| \text { and (2) }\left|\frac{k^{3} v^{6}}{27}\right| \gg\left|\frac{v^{2} \beta^{2}}{4}\right| \text {. }
$$

Since $k=(m+\rho) / 4 \rho$ where $m$ is the mass of the pipe per unit length and $\rho$ is the mass of fluid per unit length, case (1) corresponds to pipes with (a) low fluid flow velocity (small $v$ ), (b) thin pipe walls (small $k$ resulting from small $m$ ), or (c) a combination of relatively low flow velocity and relatively thin pipe walls. Case (2) corresponds to pipes with $\left(\mathrm{a}^{\prime}\right)$ high fluid flow velocity (large $v$ ), $\left(\mathrm{b}^{\prime}\right)$ thick pipe walls (large $k$ resulting from large $m$ ), or $\left(c^{\prime}\right)$ a combination of high fluid velocity and relatively thick pipe walls. The data given by Ashley and Haviland [7] for the Trans-Arabian Pipeline show that

$$
\left|\frac{k^{3} v^{6}}{27}\right| \sim 3 \times 10^{-7}\left|\frac{v^{2} \beta^{2}}{4}\right| ;
$$

thus pipelines of practical interest will clearly be covered by case (1), the low velocitythin wall case. It is important to note that for any particular pipe problem, both geometry and fluid velocity must be specified before the appropriate special case can be selected. The low velocity-thin wall case can be used only if $4 k^{3} v^{4} / 27 \ll \pi^{4}$ for the particular pipe under study. 
In the low velocity-thin pipe wall case, $\left|k^{3} v^{6} / 27\right| \ll\left|v^{2} \beta^{2} / 4\right|$, the roots of the cubic are: $\alpha_{2}=(\beta v)^{1 / 3} e^{i x / 6}, \alpha_{3}=(\beta v)^{1 / 3} e^{i 5 x / 6}$, and $\alpha_{4}=(\beta v)^{1 / 3} e^{i 9 x / 6}$, corresponding to the differential equation

$$
u^{\mathrm{IV}}+i v \beta^{\mathrm{I}}-\beta^{2} u=0 .
$$

In the high velocity-thick pipe wall case, $\left|k^{3} v^{6} / 27\right| \gg\left|v^{2} \beta^{2} / 4\right|$, the roots of the cubic are: $\alpha_{2}=0, \alpha_{3}=i k^{1 / 2} v, \alpha_{4}=i k^{1 / 2} v$, corresponding to the differential equation

$$
u^{\mathrm{IV}}+k v^{2} u^{\mathrm{II}}-\beta^{2} u=0 .
$$

The rest of this paper will be primarily concerned with these two special cases.

4. Low velocity-thin pipe walls. For pipes with thin walls and low fluid velocities we have

$$
\left|4 \frac{k^{3} v^{4}}{27}\right| \ll\left|\beta^{2}\right|
$$

so that the roots of the cubic $\alpha^{3}+k v^{2} \alpha-i v \beta=0$ are:

$$
\begin{aligned}
& a=(\beta v)^{1 / 3} e^{i \pi / 6}, \\
& b=(\beta v)^{1 / 3} e^{i 5 \pi / 6}, \\
& c=(\beta v)^{1 / 3} e^{i \theta \pi / 6} .
\end{aligned}
$$

Most pipelines of practical interest will be covered by this case. For low velocities we expect $\beta \sim \beta_{0}$ where $\beta_{0}=\pi^{2}$, the lowest eigenvalue for a beam with simply supported ends. If we define $\left|4 k^{3} v^{4} / 27\right| \ll\left|\beta^{2}\right|$ to mean that $\left|4 k^{3} v^{4} / 27\right| \leq(.01) \pi^{2}$, we can compute for a given pipe (i.e., a given value of $k$ ) the maximum dimensionless flow velocity for which this case is valid. These results are given in Table I. The Trans-Arabian Pipeline

TABLE I

Maximum permissible $v$ for application of low velocity-thin wall case

\begin{tabular}{l|l|l|l|l|l|l|l|l|l}
\hline$k$ & 0.3 & 0.4 & 0.5 & 0.6 & 0.8 & 1.0 & 2.0 & 4.0 & 10.0 \\
\hline$v$ & 4.0 & 3.2 & 2.7 & 2.4 & 1.9 & 1.6 & 0.95 & 0.56 & 0.29 \\
\hline
\end{tabular}

[7] has $k \sim 0.3$ and $v=0.15$; clearly the low velocity-thin wall case is applicable. Green's function can be written:

$$
\begin{aligned}
& G_{1}(x, y, \beta)=\left(b_{1}+c_{1}\right)+\left(b_{2}+c_{2}\right) e^{a x}+\left(b_{3}+c_{3}\right) e^{b x}+\left(b_{4}+c_{4}\right) e^{c x}, \\
& G_{2}(x, y, \beta)=b_{1}+b_{2} e^{a x}+b_{3} e^{b x}+b_{4} e^{c x}, \quad 0 \leq x<y \leq 1,
\end{aligned}
$$

where $c_{i}=a_{i}-b_{i}, i=1, \cdots 4$, and where $c_{i}(y, \beta)$ are determined by the continuity of $G, \partial G / \partial x$, and $\partial^{2} G / \partial x^{2}$ and the jump discontinuity of $\partial^{3} G / \partial x^{3}$ at $x=y$. We note that the determinant of the coefficients of the equations used to determine $c_{i}$, $i=1, \cdots 4$, is the Wronskian determinant 


$$
W=a b c(a-c)(b-c)(b-a) .
$$

The parameters $a, b$, and $c$ are always distinct in the low velocity-thin pipe wall case. Thus, the integral equation (2) can be written as a nonhomogeneous Volterra integral equation

$$
\begin{aligned}
u(y)+\beta^{2} \int_{0}^{y}\left(c_{1}+c_{2} e^{a x}+c_{3} e^{b x}+\right. & \left.c_{4} e^{c x}\right) u(x) d x \\
& =-\beta^{2} \int_{0}^{1}\left(b_{1}+b_{2} e^{a x}+b_{3} e^{b x}+b_{4} e^{c x}\right) u(x) d x,
\end{aligned}
$$

where clearly the Volterra operator is well behaved as a function of $\beta$. In the case of a simply supported pipe, $u(0)=u(1)=u^{\mathrm{II}}(0)=u^{\mathrm{II}}(1)=0$, the functions $b_{i}, i=1, \cdots 4$, are determined from the boundary conditions:

$$
\begin{aligned}
b_{1}+b_{2}+b_{3}+b_{4} & =-\left(c_{1}+c_{2}+c_{3}+c_{4}\right)=C(y), \\
b_{1}+b_{2} e^{a}+b_{3} e^{b}+b_{4} e^{c} & =0, \\
b_{2} a^{2}+b_{3} b^{2}+b_{4} c^{2} & =-\left(c_{2} a^{2}+c_{3} b^{2}+c_{4} c^{2}\right)=D(y), \\
b_{2} a^{2} e^{a}+b_{3} b^{2} e^{b}+b_{4} c^{2} e^{c} & =0 .
\end{aligned}
$$

Thus, the integral on the right of (4) naturally assumes the form

$$
\int_{0}^{1}\left[\frac{C(y) E(x)}{\Delta}+\frac{D(y) F(x)}{\Delta}\right] u(x) d x
$$

where

$$
\begin{gathered}
E(x)=b^{2} c^{2}\left(e^{b}-e^{c}\right)\left(e^{a x}-e^{a}\right)+a^{2} c^{2}\left(e^{c}-e^{a}\right)\left(e^{b x}-e^{b}\right)+a^{2} b^{2}\left(e^{a}-e^{b}\right)\left(e^{c x}-e^{c}\right), \\
F(x)=e^{a x}\left[b^{2} e^{b}\left(e^{c}-1\right)-c^{2} e^{c}\left(e^{b}-1\right)\right]+e^{b x}\left[c^{2} e^{c}\left(e^{a}-1\right)-a^{2} e^{a}\left(e^{c}-1\right)\right] \\
\quad+e^{c x}\left[a^{2} e^{a}\left(e^{b}-1\right)-b^{2} e^{b}\left(e^{a}-1\right)\right]-\left[\left(b^{2}-c^{2}\right) e^{b+c}+\left(c^{2}-a^{2}\right) e^{a+c}+\left(a^{2}-b^{2}\right) e^{a+b}\right],
\end{gathered}
$$

and

$$
\begin{aligned}
\Delta=a^{2} b^{2}\left[\left(e^{a}+e^{-a}\right)-\left(e^{b}+e^{-b}\right)\right]+a^{2} c^{2}\left[\left(e^{c}+e^{-c}\right)-\right. & \left.\left(e^{a}+e^{-a}\right)\right] \\
& +b^{2} c^{2}\left[\left(e^{b}+e^{-b}\right)-\left(e^{c}+e^{-c}\right)\right] .
\end{aligned}
$$

Eq. (4) considered as a nonhomogeneous Volterra integral equation has the solution

$$
\begin{aligned}
u(y)= & -\beta^{2} \int_{0}^{1}\left\{\frac{C(y) E(x)}{\Delta}+\frac{D(y) F(x)}{\Delta}\right\} u(x) d x \\
& +\beta^{2} \int_{0}^{y} H(y, z, \beta)\left\{-\beta^{2} \int_{0}^{1}\left(\frac{C(z) E(x)}{\Delta}+\frac{D(z) F(x)}{\Delta}\right) u(x) d x\right\} d z .
\end{aligned}
$$

Eq. (5) can be rewritten as a homogeneous Fredholm integral equation with degenerate kernel, $\sum_{i=1}^{2} X_{i}(x, \beta) Y_{i}(y, \beta)$ :

$$
\begin{aligned}
u(y)= & -\beta^{2} \int_{0}^{1} \frac{E(x)}{\Delta}\left[C(y)+\beta^{2} \int_{0}^{y} H(y, z, \beta) C(z) d z\right] u(x) d x \\
& -\beta^{2} \int_{0}^{1} \frac{F(x)}{\Delta}\left[D(y)+\beta^{2} \int_{0}^{y} H(y, z, \beta) D(z) d z\right] u(x) d x .
\end{aligned}
$$


Here $H(y, z, \beta)$ is the resolvent kernel for $\widetilde{G}_{v}(y, x, \beta)=G_{v}(x, y, \beta)$ where

$$
\begin{aligned}
G_{\diamond}(x, y, \beta) & =0, \quad 0 \leq y<x \leq 1, \\
& =c_{1}+c_{2} e^{a x}+c_{3} e^{b x}+c_{4} e^{c x}, \quad 0 \leq x<y \leq 1 .
\end{aligned}
$$

and

$$
\begin{aligned}
& c_{1}=\frac{b c(b-c)-a c(a-c)-a b(b-a)}{W}, \quad c_{2}=\frac{-b c(b-c) e^{-a \nu}}{W}, \\
& c_{3}=\frac{a c(a-c) e^{-b \nu}}{W}, \quad c_{4}=\frac{a b(b-a) e^{-c \nu}}{W} .
\end{aligned}
$$

The eigenvalue equation of (6) is

$$
1+\beta^{2}\left(a_{11}+a_{22}\right)+\beta^{4}\left(a_{11} a_{22}-a_{21} a_{12}\right)=0,
$$

where

$$
a_{h k}=\int_{0}^{1} X_{h}(x, \beta) Y_{k}(x, \beta) d x .
$$

Only the first few terms of the eigenvalue equation expanded as a power series in $\beta$ are needed to obtain lower bounds for the first eigenvalue using Spiegel's formulae [11]. In order to obtain all of the terms of the eigenvalue equation of order $\beta^{4}$, the terms of $a_{11}$ and $a_{22}$ of order $\beta^{2}$ and the constant terms of $a_{12}$ and $a_{21}$ were calculated from the series expansions of $C(y), D(y), E(x)$, and $F(x)$ and inserted in (7):

$$
1+\beta^{2}\left(-\frac{1}{90} \frac{W}{\Delta}\right)+\beta^{4}\left[\left(\frac{4}{3} \frac{v^{2}}{11 !}-\frac{100}{10 !}\right) \frac{W}{\Delta}+\frac{132}{10 !} \frac{W^{2}}{\Delta^{2}}\right]+O\left(\beta^{6}\right)=0 .
$$

When $v=0, \Delta=W$ and (8) becomes

$$
1+\alpha_{1} \beta^{2}+\alpha_{2} \beta^{4}+O\left(\beta^{6}\right)=0
$$

where

$$
\alpha_{1}=-1 / 90 \text { and } \alpha_{2}=32 / 10 \text { !. }
$$

Application of Spiegel's first and second formulae [11] yields:

$$
\sum_{h=1}^{\infty} \frac{1}{\left(\beta_{h}\right)^{2}}=-\alpha_{1}=\frac{1}{90},
$$

and

$$
\sum_{h=1}^{\infty} \frac{1}{\left(\beta_{h}\right)^{4}}=\alpha_{1}^{2}-2 \alpha_{2}=\frac{1}{9450} .
$$

It is well known that the eigenvalues, $\beta_{h}$, for a simply supported beam are $(h \pi)^{2}, h=$ $1,2, \cdots$. In addition, it is well known $[12$, pp. 19, 27] that

$$
\sum_{h=1}^{\infty} \frac{1}{(h \pi)^{4}}=\frac{1}{90} \text { and } \sum_{h=1}^{\infty} \frac{1}{(h \pi)^{8}}=\frac{1}{9450} .
$$

Thus, when $v=0$, application of Spiegel's formulae to (8) gives correct results. Lower bounds for $\beta_{1}$ obtained from (10a) and (10b), respectively, are 


$$
\beta_{1} \geq \sqrt{ } 90=9.487 \text { and } \beta_{1} \geq \sqrt[4]{ } 9450=9.859 .
$$

The exact value of $\beta_{1}$ is 9.8696 which is very closely bounded by the result from (10b). When $v \neq 0$,

$$
\Delta=W\left(1-\frac{2}{8 !} v^{2} \beta^{2}+\frac{2}{14 !} v^{4} \beta^{4}+O\left(\beta^{6}\right)\right) .
$$

Application of Spiegel's first formula to (11) shows that the first zero of $\Delta$ will occur at $(v \beta)^{2} \geq 8 ! / 2$. Since $\Delta \neq 0$ in the low velocity-thin pipe wall case, (8) can be written:

$$
\begin{aligned}
1-\beta^{2}\left[\frac{8(56)+4 v^{2}}{8 !}\right]+\beta^{4}\left[\frac{32}{10 !}+v^{2}\left(\frac{16}{6 ! 8 !}\right.\right. & \left.+\frac{4}{3(11 !)}\right) \\
& \left.+v^{4}\left(\frac{4}{14 !}+\frac{4}{8 ! 8 !}\right)\right]+O\left(\beta^{6}\right)=0 .
\end{aligned}
$$

Spiegel's first and second formulae yield the following lower bounds for $\beta_{1}$ :

$$
\begin{aligned}
& \beta_{1} \geq\left[\frac{90}{1+.0089 v^{2}}\right]^{1 / 2}, \\
& \beta_{1} \geq\left[\frac{9450}{1+.0098 v^{2}+.000183 v^{4}}\right]^{1 / 4} .
\end{aligned}
$$

The lower bounds given by (13a) and (13b) are analytic functions of a real variable $v(v \geq 0)$ which can be used to bound $\beta_{1}$ for all velocities for which the low velocity-thin pipe wall case is valid. From (12) and $(13 \mathrm{a}, \mathrm{b})$ it is clear that $\beta_{1}$ is real and decreases very slightly as $v$ increases. The maximum dimensionless velocity for which (13a) and (13b) are valid is restricted by the parameter $k$, but the lower bound does not depend on $k$ explicitly. Thus, for sufficiently small dimensionless velocities all pipes will show the same decrease in $\beta_{1}$ for a given dimensionless velocity. Handelman's low velocity results [8] also show that $\beta_{1}$ decreases as $v$ increases; however, his perturbation procedure indicates a strong direct dependence of $\beta_{1}$ on $k$, namely:

$$
\beta_{1}=\pi^{2}-\left(\frac{k}{2}+\frac{1}{16}\right) \nu^{2} .
$$

Fig. 1 is a graph of $\beta_{1}$ versus $v$ showing the lower bounds calculated from (13a) and (13b), the perturbation estimates calculated from (14) for pipes with $k=0.4$ and $k=1.0$, and experimental data given by Long [6] for a simply supported pipe with $k=0.4$. Fig. 1 also show's a lower bound for $\beta_{1}$ calculated by neglecting the $v^{4}$ term in $(13 b)$ :

$$
\beta_{1} \geq\left[\frac{9450}{1+.0098 v}\right]^{1 / 4} \text {. }
$$

In the important case of $k=0.4$, it is clear from Fig. 1 that both (13b) and (13c) provide better lower bounds for the experimental data than that provided by the perturbation estimate (14). (In this case the perturbation estimate apparently is also a lower bound but this is not known a priori.) In particular (13c), which depends only on $v^{2}$, provides a very accurate lower bound for experimental values of $\beta_{1}$ for much larger values of $v$ than does (14), which also depends on $v^{2}$. Previous experience [13], [14] has shown that 


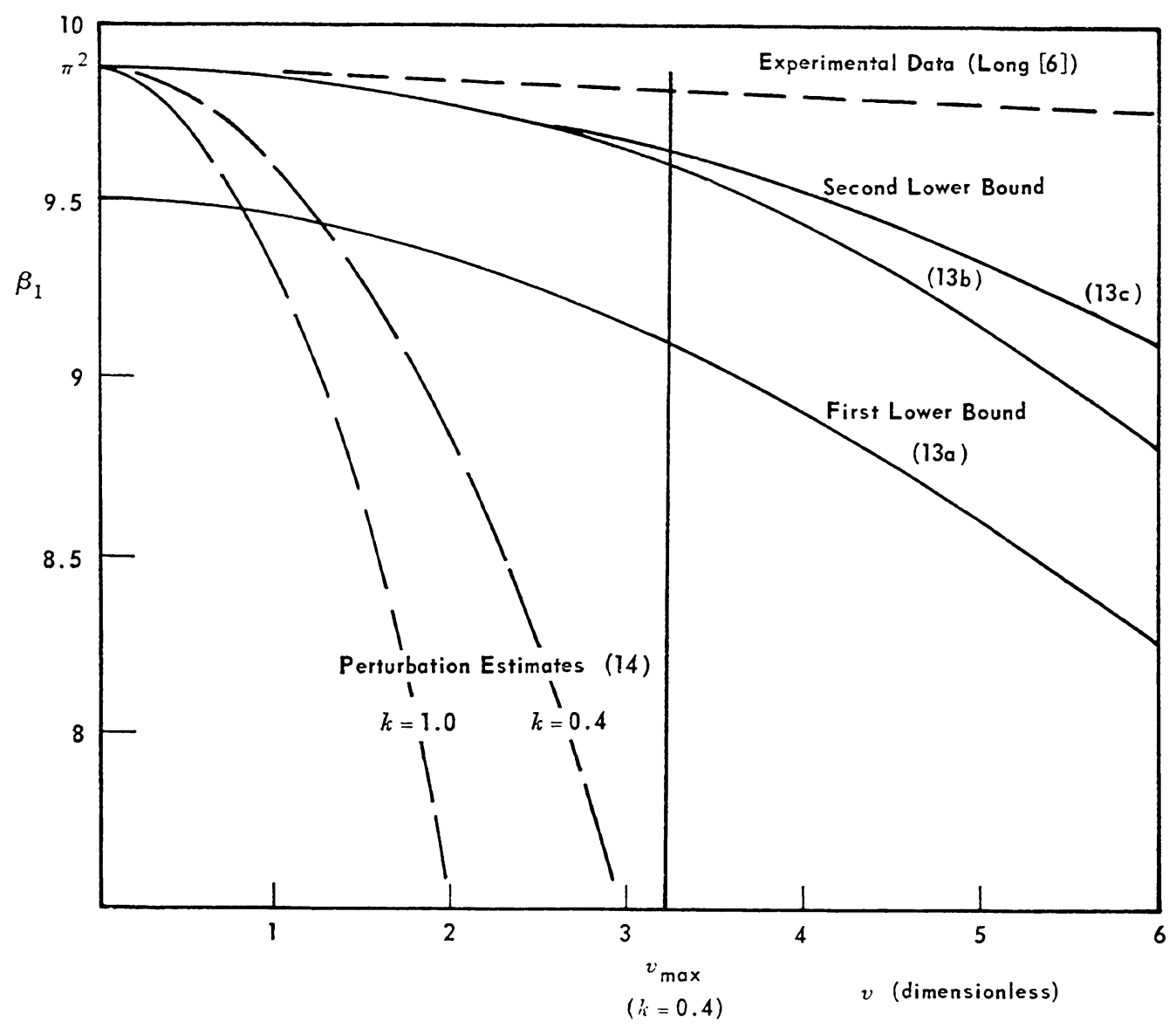

FIG. 1. The first eigenvalue $\beta_{1}$ plotted against dimensionless velocity $v$.

for problems with well-separated eigenvalues the bounds obtained by Spiegel's second formula are generally accurate to two more decimal places than those obtained from Spiegel's first formula. This is shown by comparison of the approximations to $\pi^{2}$ given by (10a) and (10b). The bound on $\beta_{1}$ given by (13b) can be further improved by calculating the terms of order $\beta^{6}$ and applying Spiegel's third formula [11]. These calculations are not prohibitively difficult and can be expected to give extremely accurate lower bounds for $\beta_{1}$.

5. High velocity-thick pipe walls. In this case Green's function is independent of $\beta$ and is given by:

$$
\begin{array}{ll}
G_{1}(x, y)=a_{1}+a_{2} x+a_{3} e^{\alpha x}+a_{4} e^{-\alpha x}, & 0 \leq x<y \leq 1, \\
G_{2}(x, y)=b_{1}+b_{2} x+b_{3} e^{\alpha x}+b_{4} e_{-}^{-\alpha x}, & 0 \leq y<x \leq 1,
\end{array}
$$

where $\alpha=i k^{1 / 2} v$ and the eight coefficients are uniquely determined. Since the operator $L[u]=u^{\mathrm{IV}}+k v^{2} u^{\mathrm{II}}$ with boundary conditions, $u(0)=u(1)=u^{\mathrm{II}}(0)=u^{\mathrm{II}}(1)=0$, is self-adjoint, Green's function will be symmetric and $\beta_{h}^{2}$ will be real for each $h$ [9]. Green's function is 


$$
\begin{array}{ll}
G_{1}(x, y)=\frac{-x(1-y)}{\alpha^{2}}+\frac{1}{\alpha^{3}} \frac{\sinh \alpha(1-y) \sinh \alpha x}{\sinh \alpha}, & 0 \leq x<y \leq 1, \\
G_{2}(x, y)=\frac{-y(1-x)}{\alpha^{2}}+\frac{1}{\alpha^{3}} \frac{\sinh \alpha(1-x) \sinh \alpha y}{\sinh \alpha}, & 0 \leq y<x \leq 1,
\end{array}
$$

where $G(x, y)$ is singular when $\alpha=i n \pi$; i.e., when $k^{1 / 2} v=n \pi, n=1,2, \cdots$. It is clear that Green's function remains symmetric for $k v^{2}>\pi$ so that the eigenvalues, $\beta_{h}^{2}$, of the integral equation

$$
u(y)=-\beta^{2} \int_{0}^{1} G(y, x) u(x) d x
$$

cannot change from real to complex at this point. Thus, $\beta_{h}$ will either be real or pure imaginary for all $h$. For arbitrary $\alpha, \alpha \neq i n \pi$, the hypotheses of Mercer's Theorem $[9$, p. 125] are satisfied and

$$
\sum_{h=1}^{\infty} \frac{1}{\left(\beta_{h}\right)^{2}}=-\int_{0}^{1} G(x, x) d x=\frac{\left(3-k v^{2}\right)-3 \cot \left(k^{1 / 2} v\right)}{6 k^{2} v^{4}} .
$$

For $k^{1 / 2} v$ near $\pi$,

$$
\sum_{1}^{\infty} \frac{1}{\left(\beta_{h}\right)^{2}} \sim \frac{-3 \cot \left(k^{1 / 2} v\right)}{6 k^{2} v^{4}}
$$

When $k^{1 / 2} v \rightarrow \pi-, \sum 1 / \beta_{h}^{2}>0$ while when $k^{1 / 2} v \rightarrow \pi+, \sum 1 / \beta_{h}^{2}<0$. Thus, $\beta_{h}$ is real for $v<\pi k^{-1 / 2}$ and at least some $\beta_{h}$ are pure imaginary for $v>\pi k^{-1 / 2}$. These results agree with Handelman's perturbation results [8]. For pipes of practical interest $(k=0.4)$, we expect unstable vibration at $v_{\text {critical }}=\pi k^{-1 / 2}=5.0$ (dimensionless). This result conflicts with experimental data given by Long [6] which show stable vibration for pipes with $k=0.4$ up to $v=10.0$ (dimensionless). We note that (1) was derived under the assumptions of small transverse deflection [5] and nondistortion of the cross-section of the pipe [10]. It would appear that at high velocities these assumptions are no longer justified and that (1) fails to provide a good description of the transverse vibration of the tube.

If Green's function (15) is written in the "natural" unsymmetric form discussed in Sec. $4,(16)$ can be written as a nonhomogeneous Volterra equation of the convolution type which can be solved by means of the Laplace transform [9]. Thus (16) becomes:

$$
\begin{aligned}
u(y)+\beta^{2} \int_{0}^{y}\left[\frac{1}{\alpha^{2}}(y-x)-\frac{1}{\alpha^{3}}\right. & \sinh \alpha(y-x)] u(x) d x \\
& =-\beta^{2} \int_{0}^{1}\left[b_{1}+b_{2} x+b_{3} e^{\alpha x}+b_{4} e^{-\alpha x}\right] u(x) d x .
\end{aligned}
$$

The Laplace transform of $u, \mathfrak{L}[u]$ is:

$$
\mathscr{L}[u]=\frac{\beta^{2}}{\left(s^{2}\left(s^{2}-\alpha^{2}\right)-\beta^{2}\right)}\left\{\left(s^{2}-\alpha^{2}\right) \int_{0}^{1} \frac{(1-x)}{\alpha^{2}} u(x) d x-s^{2} \int_{0}^{1} \frac{\sinh \alpha(1-x)}{\alpha^{2} \sinh \alpha} u(x) d x\right\},
$$

and the inverse Laplace transform of (19) is a homogeneous Fredholm integral equation with degenerate kernel which depends on the four roots, $a, b, c$, and $d$, of the quartic 
equation $s^{4}-s^{2} \alpha^{2}-\beta^{2}=0$. Thus it is possible to obtain the exact eigenvalue equation of (16):

$$
1-\beta^{2}\left(a_{11}+a_{22}\right)+\beta^{4}\left(a_{11} a_{22}-a_{12} a_{21}\right)=0,
$$

where

$$
\begin{aligned}
& a_{11}=\frac{1}{\alpha^{2}\left(a^{2}-c^{2}\right)}\left\{\frac{\left(a^{2}-\alpha^{2}\right)}{a^{3}}(\sinh a-a)-\frac{\left(c^{2}-\alpha^{2}\right)}{c^{3}}(\sinh c-c)\right\}, \\
& a_{12}=\frac{1}{\alpha^{2}\left(a^{2}-c^{2}\right)}\left\{\frac{(\sinh a-a)}{a}-\frac{(\sinh c-c)}{c}\right\}, \\
& a_{22}=\frac{1}{\alpha^{2}\left(a^{2}-c^{2}\right)}\left\{\frac{a^{2}}{\left(a^{2}-\alpha^{2}\right)}\left(a-\frac{\alpha \sinh a}{\sinh \alpha}\right)-\frac{c^{2}}{\left(c^{2}-\alpha^{2}\right)}\left(c-\frac{\alpha \sinh c}{\sinh \alpha}\right)\right\}, \\
& a_{21}=\frac{1}{\alpha^{2}\left(a^{2}-c^{2}\right)}\left\{\left(a-\frac{\alpha \sinh a}{\sinh \alpha}\right)-\left(c-\frac{\alpha \sinh c}{\sinh \alpha}\right)\right\} .
\end{aligned}
$$

and where

$$
\begin{aligned}
& a=-b=-\frac{1}{2}\left(\alpha^{2}+2 i \beta\right)^{1 / 2}-\frac{1}{2}\left(\alpha^{2}-2 i \beta\right)^{1 / 2}, \\
& c=-d=-\frac{1}{2}\left(\alpha^{2}+2 i \beta\right)^{1 / 2}+\frac{1}{2}\left(\alpha^{2}-2 i \beta\right)^{1 / 2} .
\end{aligned}
$$

Since $a_{22}$ and $a_{21}$ are singular when $\sinh \alpha=0$, i.e., when $k^{1 / 2} v=n \pi, n=1,2, \cdots$; the eigenvalue equation (20) will be satisfied if and only if

$$
\lim _{\alpha \rightarrow i \pi} \beta^{2}=0 \text { and } \lim _{\beta^{2} \rightarrow 0} \beta^{2} a_{11}=1 .
$$

Since $\lim _{\beta \rightarrow 0} a^{2}=\alpha^{2}$, the first term of $a_{11}$ clearly approaches 0 , and since $c=-i \beta / \alpha$ for small $\beta$, and $\lim _{\beta \rightarrow 0} \sinh (-i \beta / \alpha)=0$, the second term approaches $-1 / \alpha^{2} c^{2}=1 / \beta^{2}$. Thus, $\lim _{\beta^{2} \rightarrow 0} \beta^{2} a_{11}=1$ and the eigenvalue equation is satisfied exactly by $\beta=0$ at $v=n \pi k^{-1 / 2}, n=1,2, \cdots$, and again we find that $v_{\text {critical }}=\pi k^{-1 / 2}$. Unfortunately, further analysis of (20) will not provide useful information concerning the transverse vibration of a pipe because (1) no longer provides an accurate description of the physical system in the high velocity case.

6. Arbitrary flow velocities. The method used to obtain the eigenvalue equation for the high flow velocity-thick pipe wall case can also be used, in theory, to obtain the eigenvalue equation for the general case of arbitrary flow velocity. Green's function will have the form:

$$
\begin{aligned}
& G_{1}(x, y, \beta)=\left(b_{1}+c_{1}\right)+\left(b_{2}+c_{2}\right) e^{a x}+\left(b_{3}+c_{3}\right) e^{b x}+\left(b_{4}+c_{4}\right) e^{c x}, \quad 0 \leq x<y \leq 1, \\
& G_{2}(x, y, \beta)=b_{1}+b_{2} e^{a x}+b_{3} e^{b x}+b_{4} e^{c x}, \quad 0 \leq y<x \leq 1,
\end{aligned}
$$

where $a, b$ and $c$ are roots of $\alpha^{3}+k v^{2} \alpha-i v \beta=0$ and $b_{i}$ and $c_{i}, i=1,2,3,4$, are uniquely determined by the conditions on the Green's function. As before, the integral equation can be written as a Volterra equation of the convolution type which can be solved by use of the Laplace transform. The four roots, $\lambda_{1}, \lambda_{2}, \lambda_{3}, \lambda_{4}$, of the quartic equation, $s(s+a)(s+b)(s+c)-\beta^{2}=0$, must be found in order to invert the Laplace transform. The inverted equation will be a homogeneous Fredholm integral equation with degenerate kernel consisting of four terms: 


$$
u(y)=\beta^{2} \int_{0}^{1}\left\{\sum_{i=1}^{4} Y_{i}\left(y, \beta, \lambda_{1}, \lambda_{2}, \lambda_{3}, \lambda_{4}\right) X_{i}(x, \beta)\right\} u(x) d x
$$

and eigenvalue equation of this integral equation can be written down as the determinant of a $4 \times 4$ matrix. Although general application of the method is straightforward, the most useful results will be obtained when the roots $a, b$, and $c$ are such that the roots $\lambda_{1}, \lambda_{2}, \lambda_{3}, \lambda_{4}$ are relatively simple. Although the roots of a quartic equation can always be obtained by radicals, these roots will generally be very complicated.

7. Conclusion. The integral equation methods discussed in this paper and applied to the specific nonlinear eigenvalue problem which describes the transverse vibrations of a tube containing flowing fluid are quite general. Green's function for a fourth order differential operator $L_{x, \beta}$ which is an entire function of $\beta$ can always be written:

$$
\begin{aligned}
& G_{1}(x, y, \beta)=\sum_{i=1}^{4}\left[b_{i}(y, \beta)+c_{i}(y, \beta)\right] u_{i}(x, \beta), \quad 0 \leq x<y \leq 1, \\
& G_{2}(x, y, \beta)=\sum_{i=1}^{4} b_{i}(y, \beta) u_{i}(x, \beta), \quad 0 \leq y<x \leq 1,
\end{aligned}
$$

where $u_{i}(x, \beta)$ are a fundamental set for the adjoint operator, $\bar{L}_{x, \beta}$. This means that the differential equation, $L_{x, \beta}[u]+\beta^{2}[u]=0$, and appropriate boundary conditions can be written as a nonhomogeneous Volterra integral equation

$u(y)-\beta^{2} \int_{0}^{y}\left[\sum_{i=1}^{4} c_{i}(y, \beta) u_{i}(x, \beta)\right] u(x) d x=\beta^{2} \int_{0}^{1}\left[\sum_{i=1}^{4} b_{i}(y, \beta) u_{i}(x, \beta)\right] u(x) d x$.

Since the denominator of $c_{i}(y, \beta)$ is the Wronskian determinant, (22) can be solved and the first few terms of the eigenvalue equation obtained by the methods of [3]. If $L_{x, \beta}$ has constant coefficients, (22) will be a Volterra integral equation of the convolution type which can be solved by means of the Laplace transform. It is sometimes possible to obtain the exact eigenvalue equation of the problem by this method. Finally, we note that one of us has shown [15] that the eigenvalue equation obtained from the Volterra formulation discussed in this paper has the same roots as that obtained from the Fredholm formulation described previously [3], and that the lower bounds obtained from the Volterra formulation are either the same or better than those obtained according to [3].

\section{REFERENCES}

[1] L. H. Jones, The transverse vibrations of a pipe containing flowing fluid, Master's Thesis, University of Delaware, Newark, Delaware

[2] E. P. Hamilton and B. E. Goodwin, The inverse problem of the calculus of variations, in Analytic methods in mathematical physics (ed. R. P. Gilbert and R. G. Newton), Gordon and Breach, New York, 1970

[3] B. E. Goodwin, On the realization of the eigenvalues of integral equations whose kernels are entire or meromorphic in the eigenvalue parameter, SIAM J. Appl. Math. 14, 65-85 (1966)

[4] R. Iglisch, Über Linear Integralgleichungen mit vom Parameter abhängigem Kern, Math. Ann. 117, 129-139 (1939)

[5] G. W. Housner, Bending vibrations of a pipeline containing flowing fluid, J. Appl. Mech. 19, 205-208 (1952)

[6] R. H. Long, Jr., Experimental and theoretical study of transverse vibrations of a pipe containing flowing fluid, J. Appl. Mech. 22, 65-68 (1955) 
[7] H. Ashley and G. Haviland, Bending vibrations of a pipeline containing flowing fluid, J. Appl. Mech. 17, 229-232 (1950)

[8] G. H. Handelman, A note on the transverse vibration of a tube containing flowing fluid, Quart. Appl. Math. 13, 326-330 (1956)

[9] F. G. Tricomi, Integral equations, Pure and Appl. Math., vol. 5, Interscience, New York, 1957

[10] F. I. Niordson, Experimental and theoretical study of transverse vibrations of a tube containing flowing fluid, Trans. Roy. Inst. Tech., Stockholm 1953, 73 pp.

[11] M. R. Spiegel, The summation of series involving roots of transcendental equations and related applications, J. Appl. Phys. 24, 1103-1106 (1953)

[12] W. Magnus, F. Oberhettinger and R. P. Soni, Formulas and theorems for the special functions of mathematical physics, 3rd ed., Die Grundlehren der math. Wissenschaften, Band 52, Springer-Verlag, New York 1966

[13] B. E. Goodwin and W. E. Boyce, The vibrations of a random elastic string: The method of integral equations, Quart. Appl. Math. 12, 261-266 (1964)

[14] W. E. Boyce and B. E. Goodwin, Random transverse vibrations of elastic beams, J. Soc. Indust. Appl. Math. 12, 613-629 (1964)

[15] L. H. Jones, Improved lower bounds for the eigenvalues of a class of boundary value problems, SIAM J. Appl. Math. (Submitted) 